\title{
CONVENTIONAL APPROACH FOR FABRICATION OF ORBITAL PROSTHESIS: A CASE REPORT
}

\author{
Singh $T^{1^{*}}$, Mathema $S R B^{2}$, Manandhar $P^{3}$
}

\section{Affiliation}

1. Lecturer, Department of Prosthodontics, Kathmandu University School of Medical Sciences, Dhulikhel Hospital.

2. Professor, Department of Prosthodontics and Maxillofacial Prosthetics, People's Dental College and Hospital, Tribhuvan University.

3. Lecturer, Department of Prosthodontics and Maxillofacial Prosthetics, Gandaki Medical College Teaching Hospital and Research Center Pvt Ltd, Tribhuvan University.

\section{ARTICLE INFO}

Article History

Received : 24 January, 2018

Accepted : 10 August, 2018

Published： 31 August, 2018

(c) Authors retain copyright and grant the journal right of first publication with the work simultaneously licensed under Creative Commons Attribution License CC - BY 4.0 that allows others to share the work with an acknowledgment of the work's authorship and initial publication in this journal.

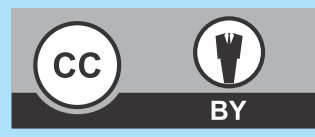

\section{CR 20}

DOI: http://dx.doi.org/10.3126/bjhs.v3i2.20969

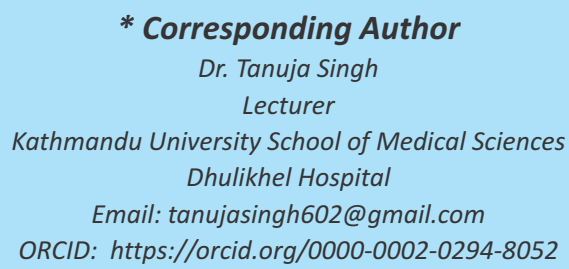

\section{Citation}

Singh T, Mathema SRB,Manandhar P. Conventional Approach for Fabrication of Orbital Prosthesis: A Case Report. BJHS 2018;3(2)6:495-499.

\section{ABSTRACT}

\section{Introduction}

Facial defect rehabilitation is a very critical task which requires personalized design of the prosthesis for each patient. Eyes are vital organ not only for vision but also for the facial expression. Disfigurement due to loss of an eye and associated structures may cause physical and psychological distress to the patient. Rehabilitating such patient with a removable orbital prosthesis is an economical and patient-friendly procedure which restores the patient's social as well as the cosmetic value. The present article describes a conventional technique for the fabrication of a spectacle retained acrylic resin orbital prosthesis providing accurate and effective rehabilitation.

\section{KEYWORDS}

Acrylic resin, facial defect, rehabilitation, orbital prosthesis. 


\section{INTRODUCTION}

Disfigurement of the face due to exenteration of an eye is a very traumatic event in a person's life not only physically but also psychologically and emotionally as face and eyes are the identities of the person. Exenteration is the surgical removal of orbital content that is eye, adnexa, and the part of the bony orbit. ${ }^{1}$ Orbital rehabilitation is a complex procedure which requires individuality in fabrication according to the patient. The earliest known examples of restorations dated back to the fourth dynasty in Egypt where rehabilitation of eye by using precious stones, copper, gold, enameled bronze, earthenware have been found in the shrunken socket of the cadaver during tomb excavation. $^{2}$

For the prosthesis to be successful various factors should be taken into consideration like color, contour, texture, biocompatibility, durability, ease of use, weight, and availability. Although several materials are available, not a single maxillofacial material fulfill all of these properties. ${ }^{3,4}$ Prosthesis for orbital defects is made from a variety of materials, such as vinyl chloride polymer, acrylic resin, medical grade silicone or polyurethane. ${ }^{5}$ Retention also plays a key role in the success of the maxillofacial prosthesis (i.e., anatomic, adhesives, and mechanical). Adhesives may cause skin allergies in long term use in some patients and for high degree of retention significant amount of supportive ingredients may be necessary to add into the adhesives. ${ }^{6}$

Though expensive, sometimes, magnets have been incorporated into the dentures for better retention of the prosthesis. ${ }^{7}$ On long term use the property of magnetism may be lost or magnets may corrode. Hence the most accepted mode for improving retention is through use of osseointegrated implants. ${ }^{8}$ But due to many reasons like inadequate bone, added surgery, extra cost for the implant and past radiation history to that area, this kind of treatment is contraindicated. ${ }^{9}$ Retention of orbital prosthesis through the use of eyeglass frames is one of the most acceptable method as it assure precise and consistent positioning of the prosthesis since the minute mistake in positioning the prosthesis will be distinctly noticed. ${ }^{10}$ Moreover, eyeglass frames offer an additional high degree of bonding, mainly to the acrylic prostheses.
This case report describes a comprehensive technique for the fabrication of spectacle retained heat activated polymethylmethacrylate (Trevalon Heat Cure, Dentsply India Pvt Ltd) orbital prosthesis.

\section{CLINICALREPORT}

A 26 year old, male patient reported to the Department of Prosthodontics and Maxillofacial Prosthetics, People's Dental College and Hospital, Kathmandu, with a chief complain of unaesthetic appearance of face due to loss of an eye and associated structure. His past history reveals exenteration of orbit five years back because of squamous cell carcinoma of the orbit. On clinical examination, the defect was large $(4 \times 5) \mathrm{cm}$ healed with the fibrotic tissue without any possible undercut for retention. Presence of healing burn injury around left alar region contraindicated complete facial impression. An acrylic orbital prosthesis attached to eyeglasses frame was planned for complete prosthetic rehabilitation of the patient.

\section{MATERIAL AND METHOD \\ Recording the Impression}

An informed consent was obtained from patient himself and explained about the procedure. Patient was relaxed and made to rest comfortably for impression making. Petrolatum was applied on eyebrows so that impression material would not stick on it and removal of impression material would be easy without any discomfort to the patient. The modeling wax (Pyrex Polykem, India) was adapted around the defect with adhesive to receive the impression material. Preparations were made to mix alginate (ZelganPlusTM, Dentsply India Pvt Ltd, Gurgaon, India) in two consistencies, first syringe consistency was mixed and poured gradually and gently until it covered the entire defect which was dispensed using a syringe and secondly tray consistency was mixed and placed over syringe consistency. Then the stapler pins were placed over the alginate to hold the dental plaster which acts as a matrix for the impression. The impression material along with dental plaster was allowed to set. The set impression was gently taken out in single piece without tearing the impression material.
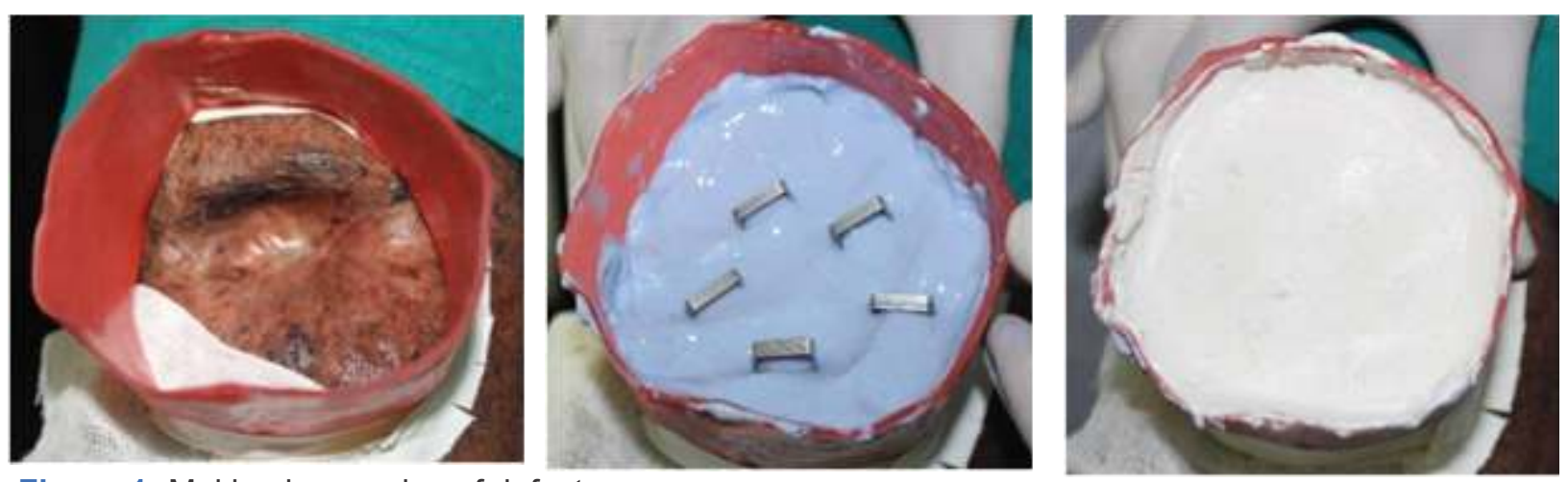

Figure 1: Making impression of defect 


\section{Wax pattern Fabrication}

The master cast was poured in type IV gypsum (Pearlstone Diestone type IV, Asian Chemicals, Gujarat India) for finer surface details and more strength. Modeling wax (Pyrex Polykem, India) was adapted over the cast and tried in patient's face. Size and orientation of the ocular part of the prosthesis was determined by taking various measurements on patient's contralateral side. Measurements from nasal bridge to the inner canthus, inner canthus to center of iris and center of iris to outer canthus of the contralateral eye were made while patient was looking and fixing the eye at a distant gaze. The measurements were recorded and transferred on to the master cast. Sclera pattern was also fabricated with the help of modeling wax and acrylized in a conventional manner. Sclera was tried in patient and iris orientation was done by asking the patient to look and fix the contralateral eye at distant gaze and by measuring the contralateral iris. Iris painting was done to match the contralateral side, completing the fabrication of the ocular portion of orbital prosthesis.

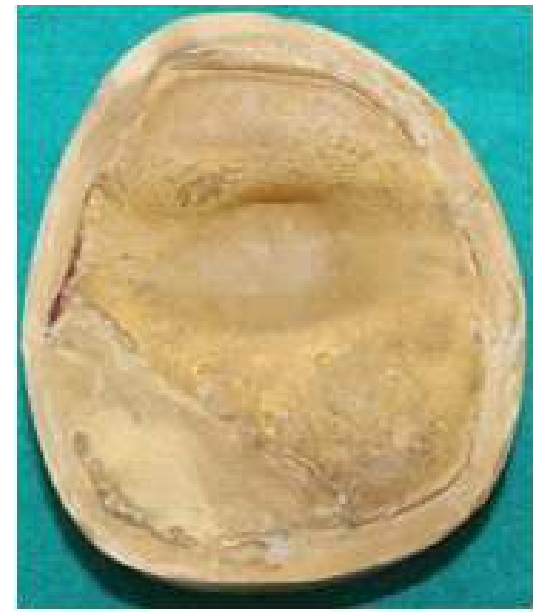

Figure 2: Working cast

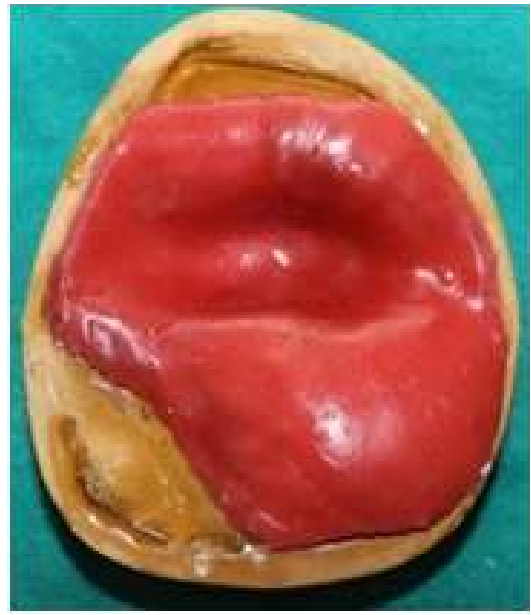

Figure 3: Wax pattern

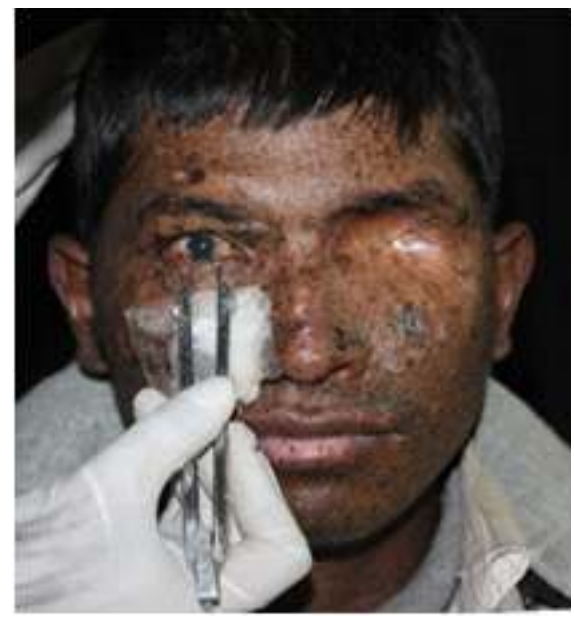

Figure 4: Iris orientation

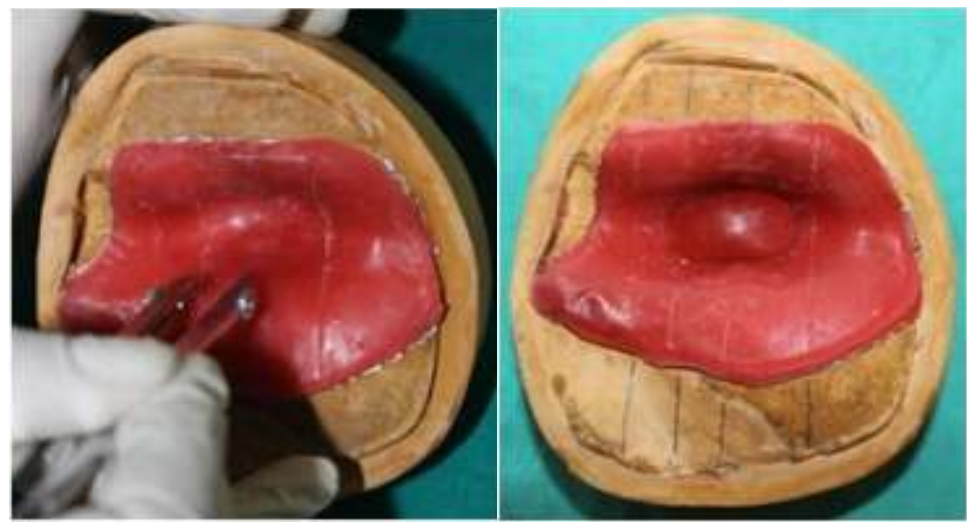

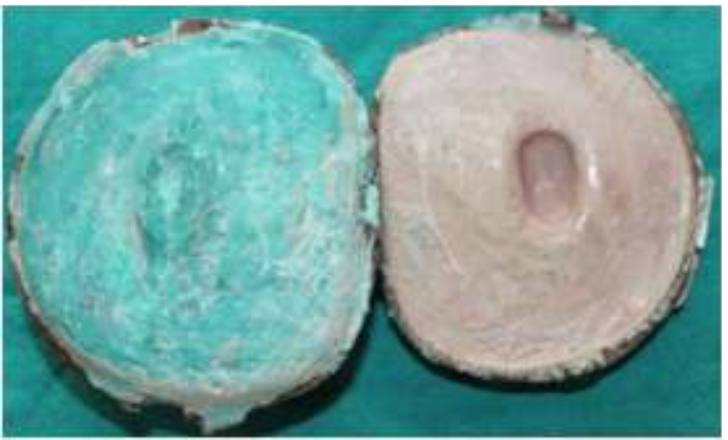

Figure 6: Dewaxing

Figure 5: Sclera orientation

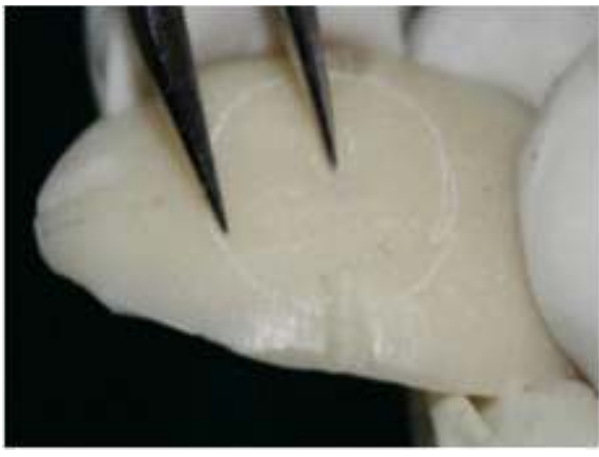

Figure 7: Iris orientation

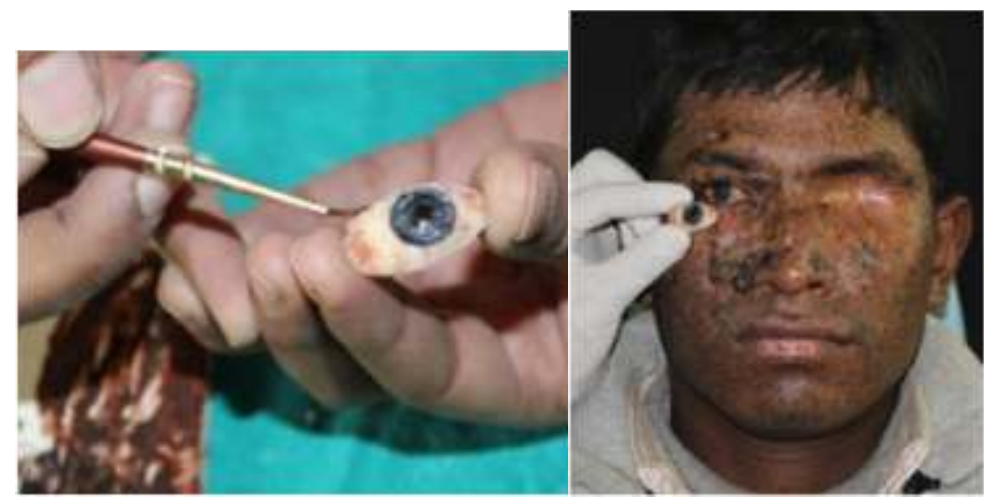

Figure 8: Eye painting 


\section{Fabrication of heat cure orbital prosthesis}

The orbital part of prosthesis was fabricated on modelling wax with the help of custom made ocular prosthesis. Following dewaxing and acrylization, final processing of the prosthesis was done. Painting of acrylic prosthesis was done to match the color to that of right side. The final prosthesis was verified for the orientation of pupil, proportion, size, shape and color of sclera as compared to the contralateral eye. The wax prosthesis along with custom made ocular prosthesis was tried on patient. To determine final position of ocular prosthesis it was first secured onto a bed of modelling wax and positioned according to measurements from contralateral eye. The anteroposterior position of the prosthesis was confirmed from profile as well as from coronal view. Eyelids and remaining portion of the prosthesis was carved and tried for final position and fit into the defect. The wax pattern with master cast was flasked and dewaxed with the wax sculpted prosthesis. Transparent heat cure acrylic resin was mixed in a porcelain jar, according to the instruction given by manufacturer. Acrylic color was and readymade eyelashes (Huda beauty eye lashes, distributed by Huda beauty LLC, Dubai) were glued on the eyelids of the acrylic prosthesis, to give lively appearance. The eyeglass frame was tried in the patient. Once patient approved eyeglass frame, the prosthesis was attached on the eyeglass frame for retention and proper orientation. Margin of the prosthesis was aligned along the border of the frame for natural appearance. The prosthesis was placed on the patient and home care instructions were given. Regular follow-up was conducted for the evaluation of the prosthesis. The patient was happy and satisfied as the prosthesis fulfilled the function and esthetic demand which made him socially presentable.

\section{DISCUSSION}

Orbital defect rehabilitation is a tedious procedure involving aesthetic element. Even a minute difference in positioning the eye and the color of the prosthesis will be markedly noticeable on the appearance of the patient. The intensive surgical procedure, a major financial burden to the patient may lead patient to seek economical prosthetic treatment options. When the reconstructive surgical procedure is

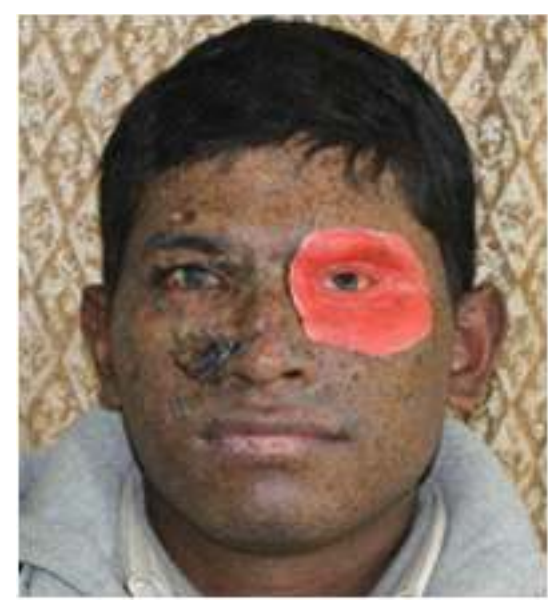

Figure 9: Try in of orbital prosthesis

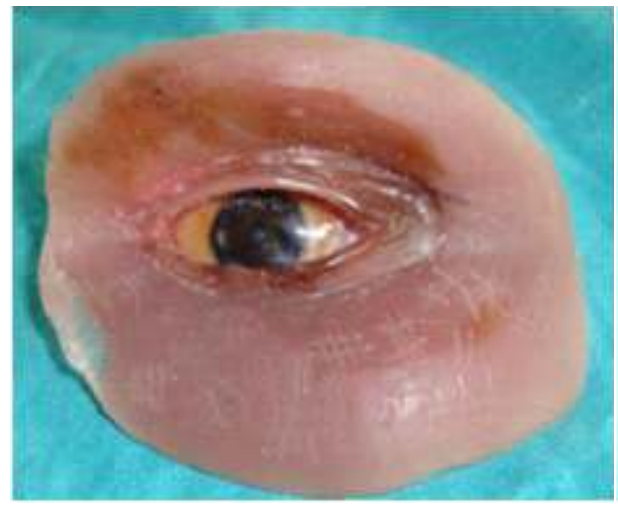

Figure 12: Prosthesis after intrinsic color

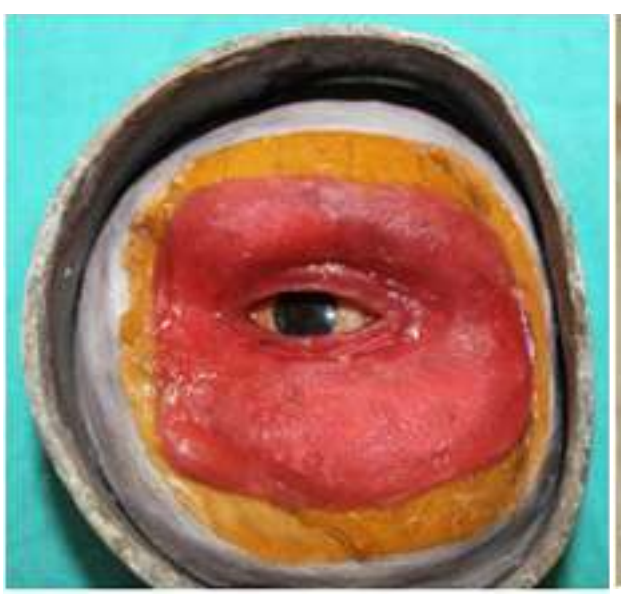

Figure 10: Flasking

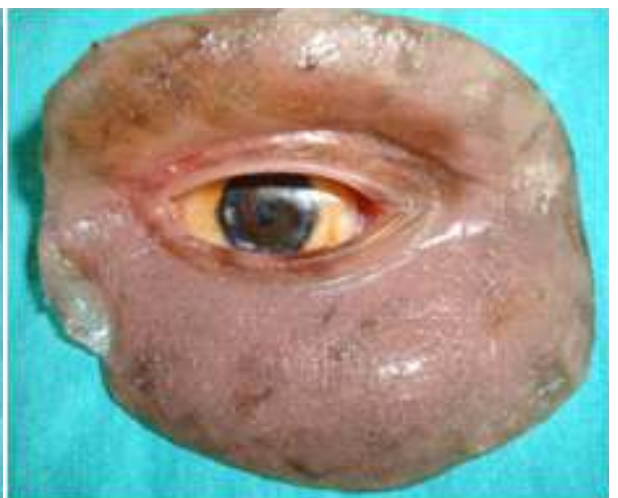

Figure 13: Prosthesis after extrinsic color

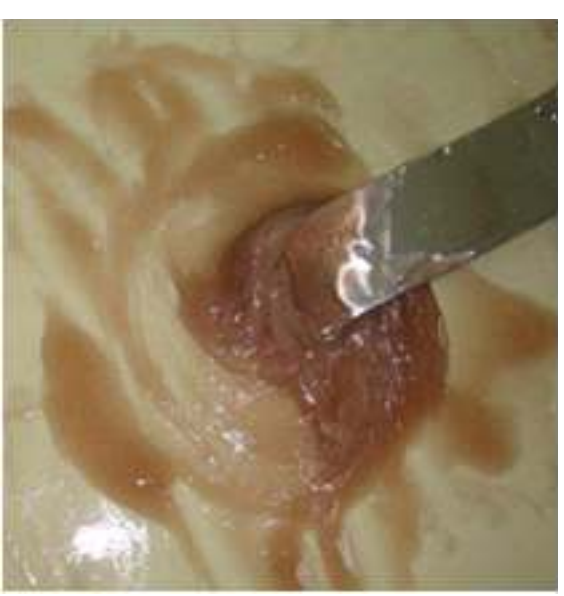

Figure 11: Coloration

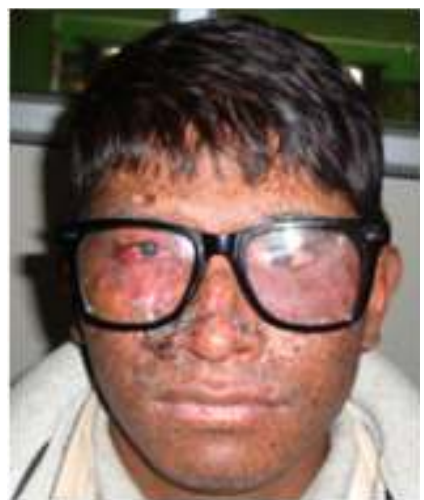

Figure 14: post-treatment photograph mixed into the base color of acrylic resin uniformly to match intrinsic stains and skin shade of patient. The colored heat cure acrylic resin was packed, bench cured, cured and bench cooled for overnight.After bench cooling, the prosthesis was retrieved, finished and polished.Natural hair from patient was glued over the eyebrow area to make eyebrows beyond the capacity of the patient, orbital prosthesis is the best alternative as it is simple, economical and does not involve any invasive procedure. ${ }^{11}$ Facial rehabilitation by the prosthesis with defect due to surgery leads to less postsurgical trauma to the patient as it boost the personality, self-image and social acceptability thus enhances the quality of life of the patient. Desirable properties of material 
for maxillofacial prosthesis comprises of elasticity, biocompatibility, moldability, longevity and ability to accept intrinsic and extrinsic colorants. ${ }^{12}$ Anatomic retentive undercuts and eyeglass frames are the most frequently used traditional method to retain orbital prostheses. ${ }^{4}$ The availability of anatomic undercut play the major role in the selection of material used for fabrication of the orbital prosthesis. Flexible material will be beneficial for the patients presenting with engageable orbital undercuts. In contrary, in cases with complete loss of orbit and without any possible engageable undercuts, the eyeglass frame retention method is the economical and convenient as eyeglass frames are easier to place in an exact, reproducible prosthesis position. The patient was treated with eyeglass frame retained acrylic resin orbital prosthesis in this case report. Acrylic prostheses attached to the eyeglass frames are much superior compared to the older methods of silicone adhesive systems, as it is more biocompatible and can be easily removed and placed in the same position. ${ }^{13}$ Acrylic resin is a durable material compared to the silicone orbital prostheses which have a relatively short lifespan (on an average of 1.5 to 2 years). ${ }^{14}$

\section{CONCLUSION}

The utilization of custom fabricated orbital prosthesis has proved to be blessing for the patient who cannot undergo extensive reconstruction surgery or afford the expensive treatment options. The acrylic orbital prosthesis presented hereis cost effective, affordable and acceptable which meet the physiologic, anatomic, functional and cosmetic requirements of the patient. In addition, the prosthesis design incorporated retention features during the function, which is the major criteria for the success of the prosthesis. This method has provided favorable results in terms of patient cosmetics, recognition, and expectations.

\section{FINANCIAL DISCLOSURE}

None

\section{REFERENCES}

1. Mishra SK, Ramesh C. Reproduction of custom made eye prosthesis maneuver: A case report. Journal of Dentistry and Oral Hygiene. 2009 Dec 31;1(5):59-63.

2. Sinha ND, Bhandari A, Gangadhar SA. Fabrication of custom ocular prosthesis using a graph grid. Pravara Med Rev. 2009 Mar;4(1):21-4.

3. Marunick MT, Harrison R, Beumer J. Prosthodontic rehabilitation of midfacial defects. Journal of Prosthetic Dentistry. 1985 Oct 1;54(4):553-60. DOI: 10.1016/0022-3913(85)90433-0.

4. Begum Z, Kola MZ, Joshi P. Analysis of the properties of commercially available silicone elastomers for maxillofacial prostheses. International Journal of Contemporary Dentistry. 2011 Aug 16;2(4).

5. Soni BW, Soni N, Bansal M. Orbital prosthesis: a novel reconstructive approach. Oral Health Dent Manag. 2014 Sep;13(3):690-3. PMID:25284538.

6. Kiat-amnuay S, Gettleman L, Khan Z, Goldsmith LJ. Effect of adhesive retention on maxillofacial prostheses. Part I: Skin dressings and solvent removers. Journal of Prosthetic Dentistry. 2000 Sep 1;84(3):335-40.doi: 10.1067/mpr.2000.109507.

7. Dumbrigue HB, Fylerb A. Minimizing prosthesis movement in a midfacial defect: a clinical report. Journal of Prosthetic Dentistry. 1997 Oct 1;78(4):341-5. doi: 10.1016/S0022-3913(97)70040-4.

8. Wolfaardt JF, Tam V, Faulkner MG, et al: Mechanical behavior of three maxillofacial prosthetic adhesive systems: a pilot project. J Prosthet Dent 1992;68:943-949. doi: 10.1016/0022-3913(92) 90556-P.
9. Arcuri $M$, Lavelle WE, Flyer $E$, et al: Prosthetic complications of extraoral implants. J Prosthet Dent 1993;69:289-29. doi: 10.1016/ 0022-3913 (93)90108-Z.

10. Gunjan P, Veena J, Swati S: A novel method for retention of an orbital prosthesis in a case with continuous maxillary and orbital defect. J Indian Prosthodont Soc 2010;10: 132-136. doi:10.1007/s13191010-0025-x.

11. Konstantinidis L, Scolozzi P, Hamedani M. Rehabilitation of orbital cavity after total orbital exenteration using oculofacial prostheses anchored by osseointegrated dental implants posed as a one-step surgical procedure. KlinMonblAugenheilkd. 2006; 223: 400-404. doi: 10.1055/s-2006-926565.

12. Jani RM, Schaaf NG. An evaluation of facial prostheses. The Journal of Prosthetic Dentistry. 1978; 39: 546-550. doi: 10.1016/S0022 3913 (78) 80191-7.

13. Dootz ER, Koran A, III, Craig RG: Physical properties of three maxillofacial materials as a function of accelerated aging. J Prosthet Dent 1994;71:379-383. doi: 10.1016/0022-3913(94)90098-1.

14. Visser A, Raghoebar GM, Oort RP, et al: Fate of implant-retained craniofacial prostheses: life span and aftercare. Int J Oral Maxillofac Implants 2008;23:89-98. PMID:18416416. 